\title{
COMPUTER-ASSISTED GRAMMAR PRACTICE FOR ORAL COMMUNICATION
}

\author{
Stephen Bodnar, Catia Cucchiarini, Helmer Strik \\ Department of Linguistics, Radboud University Nijmegen, The Netherlands \\ s.bodnar@let.ru.nl,c.cucchiarini@let.ru.nl,h.strik@let.ru.nl
}

Keywords: computer-assisted language learning, corrective feedback, grammar instruction, oral proficiency

\begin{abstract}
Gaining the ability to speak proficiently is an important goal in second language learning, and grammatical correctness is an important dimension of oral proficiency. To acquire the ability to produce grammatically correct speech in everyday conversational situations, learners must practice producing speech until they can do so with little to no concious effort. For maximum pedagogical effectiveness, practice exercises should challenge learners to produce spoken output and provide corrective feedback $(\mathrm{CF})$ on their productions so that learners may notice and correct their mistakes. In this paper, we survey the field of Intelligent Computerassisted Language Learning (ICALL) to examine the extent to which current offerings meet the pedagogical requirements for training grammatical accuracy in oral communication. Our analysis shows that few grammarfocused systems support oral practice, and that systems which do offer oral practice tend to train conversational fluency. In response to these findings, we present our position that grammar accuracy should be addressed in ICALL systems and that, in spite of technological limitations, it is possible to deploy speech technology in ICALL systems to support spoken interaction and allow individualized oral grammar practice and feedback.
\end{abstract}

\section{INTRODUCTION}

Gaining the ability to speak proficiently is an important goal in second language (L2) learning, and grammatical correctness is an important dimension of L2 proficiency (Housen and Kuiken, 2009). (Brumfit, 1984) distinguished between accuracy-oriented activities, those aimed at promoting the production of grammatically correct language, and fluency-oriented activities, those aimed at stimulating spontaneous L2 production. Exposure to the target language and usage-based learning are viewed as essential elements in Second Language Acquisition (SLA) (Ellis and Bogart, 2007). However, a considerable body of SLA research has also shown that language input and usage-based learning are not enough and that focus on linguistic form and accuracy is often required to help L2 learners achieve target-like levels of proficiency. Through instruction, output, intensive practice and corrective feedback L2 learners can acquire the ability to produce grammatically correct speech in everyday conversational situations. In general, learn- ers do not have many opportunities to engage in the kind of practice they need, namely spoken interactions where feedback is provided. In language classrooms the emphasis is typically on meaning instead of form, the difficulty level and the curriculum are not tailored to the needs and interests of each learner, and large class sizes limit the amount of practice available.

Recent developments in Intelligent ComputerAssisted Language Learning (ICALL) may provide new opportunities for offering intensive practice in speaking the target language and personalized corrective feedback. However, an analysis shows that the majority of systems that train grammar skills do not support spoken interaction, and that speechinteractive systems typically do not individualize language instruction and tend to focus on other aspects of oral proficiency such as pronunciation or conversation skills.

It is our opinion a) that grammar accuracy, as an important dimension of oral proficiency, should be addressed in ICALL systems and b) that in spite of technological limitations, it is possible to deploy 
speech technology in ICALL systems to support spoken interaction and allow individualized oral grammar practice and feedback.

This paper is organized as follows. In section 2, we draw on literature from Second Language Acquisition (SLA) research to explore the pedagogical requirements for providing sound and effective grammar practice. Section 3 employs the features presented in the previous section as desiderata for oral grammar practice to review recent advanced computer applications from ICALL. In Section 4, we present our position that pedagogically sound ICALL systems for oral grammar practice can be developed by using Automatic Speech Recognition (ASR).

\section{REQUIREMENTS FOR ORAL GRAMMAR PRACTICE}

\subsection{Literature overview}

In the field of SLA there has been considerable debate on the role of input, output, practice and corrective feedback as factors in promoting the acquisition of a second language. Proposers of the Input Hypothesis and the Natural Approach (Krashen, 1982) (Krashen and Terrell, 1983) maintain that language acquisition is driven by exposure to comprehensible language input, and that "speech (and writing) production emerges as the acquisition process progresses" (Krashen and Terrell, 1983, p. 53). In line with this reasoning, language learners should not be stimulated to produce language output because this only plays a marginal role in the acquisition process.

However, immersion studies in which language learners were exposed to huge amounts of language input have revealed that this is not enough to achieve target-like performance in productive skills (Swain and Lapkin, 1982) (Ranta and Lyster, 2007). This resulted in a renewed emphasis on the importance of output in language learning and the possible functions it may serve (Swain, 1985) (DeBot, 1996).

Relevant in this respect is the connection to skill acquisition theory in cognitive psychology (Anderson, 1982) and in particular the distinction between declarative and procedural knowledge. In this view people acquire skills first through explicit information or rules (declarative knowledge) which are then gradually transformed into internalized routines (procedural knowledge) which eventually are automatized. Output and practice play an important role in converting declarative knowledge into procedural knowledge thus promoting fluency and automaticity (De-
Bot, 1996) (Gass, 1997). An important distinction between declarative and procedural knowledge is that while declarative knowledge is generalizable, procedural knowledge is skill-specific, and in spite of claims that it is input practice that leads to acquisition (VanPatten and Cadierno, 1993), various studies have indicated a lack of transfer from receptive to productive skills, thus underlining the importance of language output and practice (DeKeyser and Sokalski, 1996) (Izumi, 2002) when it comes to learning to speak and write a second language.

Another debated issue in SLA research is the function of corrective feedback in general and the specific features, effectiveness and timing of different feedback moves (Nicholas et al., 2001). Some researchers claim that the evidence in favour of corrective feedback is insufficient, but various studies and meta-analyses (Russell and Spada, 2006) tend to indicate that, in general, corrective feedback has a positive effect on language learning. In addition, there are indications that, to be effective, corrective feedback should be unambiguous, consistent, intensive, should provide opportunities for self-repair and modified output (Tatawy, 2002), should not be too asynchronous as it is important to give feedback while the procedural "knowledge" that led to the error is still active in memory (DeKeyser, 2007) and should preferably take account of specific learner characteristics.

\subsection{Results}

The brief literature overview presented above has produced interesting results with respect to the desirable features of ICALL systems intended to help L2 learners acquire the ability to produce grammatically correct L2 speech. First, these systems should provide comprehensible input. Second, they should stimulate skill-specific output and practice. Third, they should produce accurate corrective feedback that is clear, consistent, intensive, timely, individualized and that provides opportunities for self-repair and modified output. In the following section we proceed to analyzing a number of available systems to determine to what extent they satisfy the criteria outlined above.

\section{COMPUTER SYSTEMS FOR GRAMMAR PRACTICE}

To investigate how computer applications might be able to help language learners improve the grammatical accuracy of their speech, we searched the ICALL literature (e.g., Computer Assisted Language 
Learning, ReCALL, Speech Communication, System, Language Learning and Technology, CALICO Journal, and others) for reports of ASR-enabled systems that train grammar accuracy. Finding relevant systems proved more difficult than expected (an early search yielded just one system), and as a result we relaxed search criteria to include two categories of systems: a) those that offer grammar training regardless of whether they utilize ASR (we refer to these as "accuracy-focused" systems) and b) ASR-enabled systems which implement exercises that do not focus on grammar training but which involve use of L2 grammar knowledge (we refer to these as "fluencyfocused" systems). Relaxing these criteria increased the number of relevant systems, and from these candidates we selected those that come closest to meeting the pedagogical requirements presented above.

This section provides an overview of the systems with respect to practice exercises offered, types of feedback provided, and ability to accommodate learner differences for the two categories of systems. In the accuracy-focused category, we have the German Tutor (Heift, 2001), CALLJ (Wang et al., 2009), Te Kaitito (Vlugter et al., 2009), and a collection of web-based language games from the CSAIL MIT lab (Seneff, 2007); and for fluency-focused systems, we review the TLCTS (Johnson and Valente, 2009), SPELL (Morton and Jack, 2005), CandleTalk (Chiu et al., 2007), a prototype version of Let's Go adapted for language learners (Raux and Eskenazi, 2004), and an unnamed prototype from the University of Göteborg (Bergenholtz, 2004).

\subsection{Practice activities}

We first present accuracy-focused systems before examining the potential for grammar training in systems oriented towards fluency.

Practice in the accuracy-focused systems is characterised by narrow, skill-focused exercises that develop the learner's ability to produce grammatically accurate utterances. Systems such as the German Tutor and CALLJ are good examples of accuracyfocused systems: learning is structured into different lessons that cover vocabulary and grammar items found in language learning materials (e.g. the Japanese Language Proficiency Test). Each lesson contains activities that challenge the learner to apply target language grammar rules to produce an errorfree phrase or sentence; when the learner arrives at an answer they can submit it to the program for evaluation. Activities typically resemble activities found in traditional classroom materials, such as cloze tests, and sentence-building and translation exercises.
A different approach in accuracy-focused instruction has been to provide more realistic contexts (in the sense that the exercises more closely resemble the everyday situations where the learners will be expected to use the target language to communicate). An example of this approach can be found in Te Kaitito, which implements an accuracy-focused exercise that takes the form of text-based dialogues between the learner and a virtual tutor character. The dialogues are specially designed to elicit language that requires use of target L2 forms known to be difficult.

Practice in fluency-focused systems stands in contrast to the previous group. These systems focus on helping learners to become functional in the target language, so as to be able to satisfy their everyday communicative needs, such as describing one's likes and dislikes or asking for directions. Exercises are task-based and allow the learner to converse with virtual characters in a target language. Systems differ in the richness of the simulation: in the Let's Go system, learners telephone an automated spoken dialogue system for bus information; CandleTalk and the Bergenholtz system provide virtual two-dimensional characters with whom to interact; SPELL and TLCTS place learners in a three-dimensional virtual world (navigable via a player-controlled avatar) populated with virtual characters.

With respect to training for oral communication, one aspect that differentiates some applications from others is the modality supported by the program. Many accuracy-focused systems require that the learner produce written output by using the keyboard, or through selection exercises that rely on use of the mouse. CALLJ and the collection of web-based games are notable exceptions, as they are the only accuracy-focused systems we are aware of that employ ASR to implement grammar training exercises that allow the learner to speak to the system with a microphone.

In the process of completing exercises like those presented above, learners will inevitably produce language that contains errors. Next, we examine how ICALL systems respond to errors with corrective feedback.

\subsection{Corrective feedback}

ICALL systems can help learners to reduce the frequency of errors in their speech by drawing attention to, explaining and supplying corrections for errors through the provision of automatic corrective feedback. In the context of a practice exercise, an application provides feedback by utilizing specialized language or speech technology to monitor learner output 
for errors. When errors are discovered, the system processes the error to prepare a feedback response, which can be described as a message or behaviour that communicates that one or more errors were detected and provides information to guide the learner to correct the error(s). Naturally, the details of the feedback message vary from system to system, and may be influenced by pedagogical and technical considerations.

One factor that influences feedback is the pedagogical focus of the system. In accuracy-focused systems, emphasis on the reduction of grammar errors seems to motivate system designers to use unambiguous, explicit feedback. The German Tutor and CALLJ systems use metalinguistic descriptions to provide error-specific feedback that includes explanations, such as in the example "Watch out! The verb WOHNEN and the subject ICH do not agree. ICH is singular" from the German Tutor. Other options are explicit recasts or clarification requests which omit detailed metalinguistic language and instead supply corrected versions of an utterance. An example of this kind of feedback from the Te Kaitito system is "There's a mistake in that sentence. Maybe you mean -suggested-correction-? Let's try again”.

For fluency-focused systems, we observed that there is a tendency towards implicit feedback, feedback that is less recognizably corrective and more easily integrated into the simulation. Perhaps the most simple example can be found in TLCTS, in which characters respond to errors with simple (and perhaps realistic) requests for clarification such as "Sorry, I don't understand". A second example, this time of an implicit recast from the Bergenholtz system, can be seen in the following interaction: "Student: How old years is Dirk? System: How old is Dirk? Dirk is 30 years (old)". An explanation for the tendency towards implicit feedback is that fluency-focused practice exercises are designed to be authentic and that frequent error correction with overly explicit feedback such as metalinguistic descriptions may detract from the realism of the simulation.

A second factor that influences feedback in ICALL systems is technical feasibility. Reliable performance of the language and speech processing modules that detect and classify errors is important, as poor performance may result in errors going unnoticed or the potentially worse case of errors being reported for well-formed utterances. For this reason, system developers must take the limits of ASR components into consideration when designing feedback properties of a system. The current shortage of studies investigating the reliability of corrective feedback for grammar in ASR-enabled systems prevents strong conclusions, but existing studies suggest that correc- tive feedback is more reliable in constrained practice of the type seen in accuracy-focused systems. Performance results for CALLJ suggest adequate reliability, while data for the SPELL and Let's Go systems suggest otherwise, leading the authors of the first system to state that performance is "not high enough to support precise analysis of learner errors" (Anderson et al., 2008, p.616).

In some systems, feedback properties may also be affected by the proficiency level of the learner. In the next section, we look at how feedback and other aspects of the system can be adapted to meet individual learner needs.

\subsection{Individualized instruction}

Computer systems have excellent logging capabilities which can be used to make detailed recordings of the interaction between the system and the learner. ICALL systems employ student models to organize the information they collect into data structures that can capture different information about the learner. The type of information captured in a student model varies between systems, but some of the more common types concern study activity, including lessons completed and time on task, and performance on exercises and quizzes (for examples, see TLCTS and the German Tutor). Additionally, some systems label practice exercises with metadata related to pedagogical objectives, with the benefit being that results of completed exercises can then be taken as an indication of the degree of mastery with respect to the objectives. Examples of metadata types are linguistic items, such as particular classes of words or grammatical constructions (Heift and Nicholson, 2001), or task-oriented skills the learner is expected to acquire (Johnson and Valente, 2009). More advanced student models, in addition to the above, also capture type and frequency information about errors the learner produces, such as spelling, word order, and specific grammar errors (Heift and Nicholson, 2001).

On its own, the information captured by a student model is valuable to the student and educators as a form of summative feedback which can serve as an indicator of progress towards the goals of the system. However, the most advanced systems go one step further and use this information to adapt different aspects of the system with the aim of optimizing the instruction to a learner's specific needs. For example, the German Tutor uses a student model to adjust properties of the feedback by varying the amount of detail that is included in the error message according to the learner's skill level. Other uses of the student model in this system include guiding decisions about what 
errors to provide feedback on when multiple errors are detected in learner input and generation of remedial exercises based on observed student difficulties.

Summarising our observations, we find that practice in the systems surveyed can be grouped into accuracy- and fluency-focused systems. Practice in both groups provides opportunities for written and spoken output. Feedback types differ, with grammarfocused systems use explicit feedback to point out errors to learners, while fluency-focused systems use implicit feedback forms likely to integrate well with simulated spoken exchanges. Elements of individualised instruction can be found in both groups, including adaptation of course content, selection of errors for feedback treatment and type of feedback provided, and suggestion of remedial exercises.

The purpose of the work so far has been to evaluate the suitability of current ICALL systems to provide grammar practice for oral communication. In the next section we discuss the results of this analysis and present recommendations that support our position that developing an ICALL system that provides grammar practice for oral communication is indeed possible.

\section{RESULTS AND RECOMMENDATIONS}

In section 3 we have reviewed a number of ICALL systems. This analysis has revealed that each of the systems reviewed falls short of fulfilling the requirements for optimal computer-based grammar practice for oral proficiency.

With respect to type of practice and interaction, we have observed that few systems allow the learner to speak their output and are capable of processing the learners speech to detect errors and provide detailed corrective feedback on grammatical errors, while it seems that it is exactly the combination of spoken interaction and form-focused activities with detailed corrective feedback that is particularly required to improve grammatical accuracy in L2 speaking. Findings that support the skill-based character of procedural knowledge underline the importance of speaking practice to learn to speak an L2 (DeKeyser, 2007). The attested failure of immersion students to achieve target-like grammatical accuracy in speaking emphasizes the importance of form-focused activities and feedback to draw attention to less salient linguistic features.

In spite of these findings, we are convinced that it is possible to develop ICALL systems that exhibit the desirable properties presented above. In the remainder of this section we explain how.

First of all, it is important to take account of technical limitations. ASR performs less-reliably on nonnative speech, and at the moment, best practices recommend limiting the ASR task to discriminating between a small number of candidate utterances. While this may seem a considerable limitation, it is sufficient to allow the type of focused grammar practice seen in the German Tutor and CALLJ. Previous research from our lab on ASR-based corrective feedback on L2 pronunciation has indicated that it is possible to develop systems that supply accurate feedback (Cucchiarini et al., 2009), provided that constrained and pedagogically sound exercises are developed. In this connection it is important to point out that providing accurate corrective feedback on pronunciation is more challenging than providing feedback on grammar (Morton and Jack, 2005). In more recent research we are developing technology for providing feedback on grammar (Strik et al., 2010).

As to the precise form of feedback to be used, our analysis has indicated that ICALL systems employ a variety of different techniques to draw attention to learner errors, including simple clarification requests, implicit and explicit recasts and explicit correction, with varying degrees of error-specific metalinguistic information. Studies that have examined human-provided feedback do not provide definitive answers as to which form of feedback is most effective (see section 2). Research on feedback provided by ICALL systems seems to indicate that feedback that is explicit, error-specific and indicates the location of errors result in larger learning gains than less specific types of feedback (see (Hanson, 2007) for a review; see also (Heift, 2004)). Complicating the issue is the fact that these studies refer to practice done in the typed medium. To the best of our knowledge no research exists which has looked into the effectiveness of different feedback types in oral practice with an ICALL system.

Because of the lack of conclusive findings regarding feedback effectiveness, we recommend an approach that involves developing a library of feedback techniques. This library would be useful for at least two reasons: first, it would allow researchers to conduct their own experiments to investigate the effect of different feedback types in spoken grammar practice. Second, the library could be used later as a resource for individualising instruction, as different feedback types may be more effective for different learners or exercises.

Concerning individualised instruction, we make the observation that the German Tutor, an accuracy- 
focused system which does not include ASR, possesses superior adaptability compared to other systems reviewed. The student model influences the amount of detail included in feedback messages, guides error selection when input contains multiple errors, and informs the module that generates remedial exercises, all functions that greatly enhance the adaptability of the system to individual learners and which we endorse for ASR-enabled grammar practice systems. To achieve this functionality, a system should implement a student model and supporting framework capable of capturing information regarding study activity, performance on practice exercises, and detailed error-related data, in addition to attaching metadata to practice exercises to label them with the grammar feature(s) they target.

To summarize, on the basis of our reviews of SLA literature on the one hand and of existing ICALL systems for oral grammar training on the other, we would like to stress the importance of employing ASR technology to allow spoken interaction in relatively constrained exercises in which plausible, realistic communicative contexts are created, but where the learners output is predictable and for this reason can be analyzed automatically in detail with acceptable levels of accuracy. In turn, accurate error detection through ASR constitutes the essential basis for providing skillspecific individualized instruction, practice and feedback, thus meeting SLA requirements as much as possible.

At the moment, we are in the process of developing an ICALL system in line with the recommendations above (see Figure 1). The system uses ASR in meaning-oriented question-and-answer exercises designed to elicit forms known to be problematic for Dutch L2 learners. A pilot experiment planned for the spring of 2011 will investigate the effect of computerprovided corrective feedback on grammar accuracy, as well as the reliability and accuracy of the underlying technology.

\section{ACKNOWLEDGEMENTS}

This work is part of the research program Corrective Feedback and the Acquisition of Syntax in Oral Proficiency (FASOP), which is funded by the Netherlands Organisation for Scientific Research (NWO).

\section{REFERENCES}

Anderson, J. N., Davidson, N., Morton, H., and Jack, M. A. (2008). Language learning with interac-

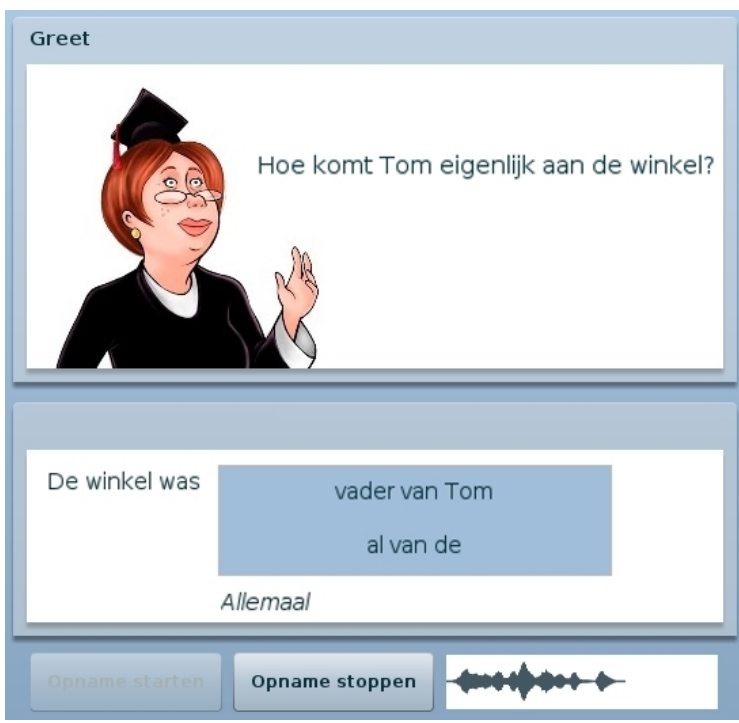

Figure 1: A screenshot of the ICALL system under development. Learners speak into a microphone to answer questions posed by a virtual tutor about a film clip. In this example, the tutor is asking 'How did Tom actually come to the store?', and the learner responds using the prompt and phrases located in the bottom half of the screen.

tive virtual agent scenarios and speech recognition: Lessons learned. Comput. Animat. Virtual Worlds, 19(5):605 - 619.

Anderson, J. R. (1982). Acquisition of cognitive skill. Psychological Review, 89:369 - 406 (37).

Bergenholtz, J. (2004). An interactionist and taskbased approach to computer-assisted language learning materials. Master's thesis, University of Götheborg.

Brumfit, C. (1984). Communicative Methodology in Language Teaching: The Roles of Fluency and Accuracy. Cambridge University Press, Cambridge.

Chiu, T.-L., Liou, H.-C., and Yeh, Y. (2007). A study of web-based oral activities enhanced by automatic speech recognition for efl college learning. Computer Assisted Language Learning, 20 (3):209 - 233 (24).

Cucchiarini, C., Neri, A., and Strik, H. (2009). Oral proficiency training in dutch 12: The contribution of asr-based corrective feedback. Speech Communication, 51 (10):853-863 (11).

DeBot, K. (1996). The psycholinguistics of the output hypothesis. Language Learning, 46:529 - 555 (26).

DeKeyser, R. (2007). Practice in a second language, chapter Introduction: Situating the con- 
cept of practice. New York: Cambridge University Press.

DeKeyser, R. M. and Sokalski, K. J. (1996). The differential role of comprehension and production practice. Language Learning, 46 (4):613-642 (29).

Ellis, N. C. and Bogart, P. S. H. (2007). Speech and language technology in education: The perspective from sla research and practice. In SLATE2007, pages 1-8 (8).

Gass, S. (1997). Input, interaction, and the second language learner. Mahwah, NJ: Lawrence Erlbaum.

Hanson, R. M. (2007). Feedback in Intelligent Computer-Assisted Language Learning and Second Language Acquisition: A study of its effect on the acquisition of French past tense aspect using an Intelligent Language Tutoring System. $\mathrm{PhD}$ thesis, University of Edinburgh.

Heift, T. (2001). Intelligent language tutoring systems for grammar practice. Zeitschrift fr Interkulturellen Fremdsprachenunterricht (online), 6(2): 1 - 15 (15).

Heift, T. (2004). Corrective feedback and learner uptake in call. ReCALL, 16(2):416-431 (15).

Heift, T. and Nicholson, D. (2001). Web delivery of adaptive and interactive language tutoring. International Journal of Artificial Intelligence in Education, 12(4):310-324 (14).

Housen, A. and Kuiken, F. (2009). Complexity, accuracy, and fluency in second language acquisition. Applied Linguistics, 30 (4):461 - 473 (13).

Izumi, S. (2002). Output, input enhancement, and the noticing hypothesis. Studies in Second Language Acquisition, 24:541 - 577 (36).

Johnson, W. L. and Valente, A. (2009). Tactical language and culture training systems: Using ai to teach foreign languages and cultures. AI Magazine.

Krashen, S. (1982). Principles and Practice in Second Language Acquisition. Oxford: Pergamon Press.

Krashen, S. D. and Terrell, T. D. (1983). The natural approach: Language acquisition in the classroom. London: Prentice Hall Europe.

Morton, H. and Jack, M. A. (2005). Scenario-based spoken interaction with virtual agents. Computer Assisted Language Learning, 18(3):171191 (20).

Nicholas, H., Lightbown, P., and Spada, N. (2001). Recasts as feedback to language learners. Language Learning, 51 (4):719-758 (39).
Ranta, L. and Lyster, R. (2007). Practice in a second language, chapter A cognitive approach to improving immersion students' oral langauge abilities: The Awareness-Practice-Feedback sequence. New York: Cambridge University Press.

Raux, A. and Eskenazi, M. (2004). Using taskoriented spoken dialogue systems for language learning: Potential, practical applications and challenges. In ICALL-2004.

Russell, J. and Spada, N. (2006). Synthesizing research on language learning and teaching, chapter The effectiveness of corrective feedback for second language acquisition: A meta-analysis of the research, pages 133-164 (31). Amsterdam: Benjamins.

Seneff, S. (2007). Web-based dialogue and translation games for spoken language learning. In Proc. of the Speech and Language Technology in Education (SLaTE) Workshop, Farmington, Pennsylvania.

Strik, H., van de Loo, J., van Doremalen, J., and Cucchiarini, C. (2010). Practicing syntax in spoken interaction: Automatic detection of syntactic errors in non-native utterances. In Proceedings of the SLaTE-2010 workshop, Tokyo, Japan.

Swain, M. (1985). Input in Second Language Acquisition, chapter Communicative competence: some roles of comprehensible input and comprehensible output in its development, pages $235-253$. Rowley MA: Newbury House.

Swain, M. and Lapkin, S. (1982). Evaluating bilingual education: A Canadian case study. Multilingual Matters Ltd., Clevedon, UK.

Tatawy, M. E. (2002). Corrective feedback in second language acquisition. Working papers in TESOL and Applied Linguistics, 2 (2):1 - 19 (19).

VanPatten, B. and Cadierno, T. (1993). Explicit instruction and input processing. Studies in Second Language Acquisition, 15 (2):225 - 243 (19).

Vlugter, P., Knott, A., McDonald, J., and Hall, C. (2009). Dialogue-based call: a case study on teaching pronouns. Computer Assisted Language Learning, 22(2):115-131 (16).

Wang, H., Waple, C. J., and Kawahara, T. (2009). Computer assisted language learning system based on dynamic question generation and error prediction for automatic speech recognition. Speech Communication, 51(10):995-1005 (20). 